\title{
Synthesis of 3-phenyl-2H,5H-pyrano[3,2-c]chromen-2-one derivatives and their antineoplastic activity
}

\author{
Lucia Kováčiková, ${ }^{a}$ Renata Gašparová, ${ }^{b *}$ Andrej Boháč, ${ }^{\text {} *}$ Marián Ďurana, ${ }^{\text {a }}$ and Margita \\ Lácováa \\ ${ }^{a}$ Department of Organic Chemistry, Comenius University, Mlynská dolina CH-2, Bratislava, 842 \\ 15, Slovak Republic \\ ${ }^{b}$ Department of Chemistry, University of SS. Cyril and Methodius, J. Herdu 2, Trnava, \\ 917 01, Slovak Republic \\ E-mail: renata.gasparova@ucm.sk; andrej.bohac@fns.uniba.sk
}

\begin{abstract}
Reaction of 4-oxo-4H-chromen-3-carbaldehydes 1 with phenylacetic acids 2 under mild conditions or microwave irradiation led to 3-phenyl-2-oxo-2H,5H-pyrano[3,2-c]chromen-5-yl acetates 3. At stronger conditions by-products 5-(2-hydroxybenzoyl)-3-phenyl-2 $H$-pyran-2-ones 4 and 5-hydroxy-2H,10a $H$-pyrano[2,3-b]chromen-2-ones 5 were also obtained. 5-Hydroxy- and 5-alkyloxy-2H,5H-pyrano[3,2-c]chromen-2-ones 6 and 7, respectively were easily prepared by reaction of 3 with water or alcohols. Twelve synthesized compounds were evaluated on their antineoplastic activities on 60 human tumour cell lines panels in NCI USA. According to the screening results 3-phenyl-2H,5H-pyrano[3,2-c]chromen-2-one was discovered as a new leading skeleton suitable for further development. Some SAR conclusions were made. Antitubuline mechanism for the most active compound $\mathbf{3} \mathbf{g}$ has been proposed.
\end{abstract}

Keywords: Pyranochromenones, phenylacetic acids, condensation, rearrangement, antineoplastic activity, microwave irradiation, tubuline inhibitors

\section{Introduction}

Synthetic exploitation of 4-oxo-4H-chromen-3-carbaldehyde 1 and its derivatives results from their reactivity towards nucleophiles. The wide variety of heterocycles can be synthesized due to the presence of three electrophilic centres in the molecule of $\mathbf{1}$ and its ability to open or retain pyran-4-one ring. ${ }^{1}$ Many of chromene derivatives exhibit significant biological activity, such as antitumour, $^{2,3}$ antimycobacterial $^{4}$ or antiviral. $^{5}$

Phenylacetic acid is versatile synthon for synthesis of a diverse set of biologically active compounds, e.g. aldose reductase inhibitors ${ }^{6}$ or hPPAR agonists. ${ }^{7}$ According to our knowledge 
only condensation of 4-oxo-4H-chromen-3-carbaldehyde $\mathbf{1}$ with phenylacetic acid $\mathbf{2}$ in pyridine catalyzed by potassium tert-butoxide has been described as the method for synthesis of 3-styryl$4 H$-chromen-4-ones. ${ }^{8}$ In connection with this, and as an extension of our studies on the synthesis, ${ }^{9,10}$ photochemical $^{11}$ and theoretical ${ }^{12}$ properties of chromene derivatives, we decided to investigate the reactions of 4-oxo- $4 H$-chromene-3-carbaldehyde 1 with substituted phenylacetic acids 2 under different conditions, to determine the products and evaluate their antineoplastic activities.

\section{Results and Discussion}

\section{Chemistry}

Several methods for the synthesis of pyrano[3,2-c]chromen-2-one derivatives were reported. $N$ (5-ethoxy-2-oxo-2H,5H-pyrano[3,2-c]chromen-3-yl)benzamides were prepared by heating of azlactones in the presence of pyridine. ${ }^{13}$ Ethyl 5-ethoxy-2-oxo-2H,5H-pyrano[3,2-c]chromene-3carboxylate was obtained by reaction of ethyl 2-cyano-3-(4-oxo-4H-chromen-3-yl)acrylate in DMF-ethanol. $^{14}$

Recently we developed convenient synthesis of 2-oxo-2H,5H-pyrano[3,2-c]chromen-5-yl acetates 3 by heating of equimolar quantities of chromene-3-carbaldehyde $\mathbf{1}$ and phenylacetic acids 2 in acetic anhydride in the presence of catalytic amount of sodium acetate or potassium carbonate. $^{15,16}$ When the mixture of $\mathbf{1}$ and $\mathbf{2}$ was heated at $60-80{ }^{\circ} \mathrm{C}$ for $2-3 \mathrm{~h}$, the acetates 3a3h were isolated as only products in $48-81 \%$ yields. When the synthesis was accomplished in microwave oven at constant $80{ }^{\circ} \mathrm{C}$, the yields of products 3 were slightly higher $(68-85 \%)$, but the duration of the reaction was considerably shorter $(30 \mathrm{~min})$. The mixture of propionic anhydride with aldehyde $\mathbf{1 a}$ and phenylacetic acid $\mathbf{2 b}$ heated at $80^{\circ} \mathrm{C}$ for $2 \mathrm{~h}$, or under microwave irradiation at $110{ }^{\circ} \mathrm{C}$ for $30 \mathrm{~min}$ both in the presence of potassium carbonate led to 3-(4fluorophenyl)-2-oxo-2H,5H-pyrano[3,2-c] chromen-5-yl propionate 3i in 80\% yield (Scheme 1). In order to examine reaction profile and the thermal stability of acetates $\mathbf{3}$, the mixture of $\mathbf{1}$ and $\mathbf{2}$ was treated at $100{ }^{\circ} \mathrm{C}$ for $12-22 \mathrm{~h}$ in $\mathrm{Ac}_{2} \mathrm{O}$. At these conditions, acetates $\mathbf{3}$ were isolated as the main products in $11-54 \%$. The by-products 5-(2-hydroxybenzoyl)-3-phenyl-2H-pyran-2-ones 4 $(1-5 \%)$ together with rearranged compounds 5-hydroxy-3-phenyl-2H,10a $H$-pyrano[2,3$b$ ]chromen-2-ones $5(2-4 \%)$ were obtained by flash chromatography of the crude reaction mixtures on $\mathrm{SiO}_{2}($ Scheme 1).

5-Hydroxy-3-phenyl-2H,10aH-pyrano[2,3-b]chromen-2-one 5a was also prepared independently by rearrangement of 3a in acetic acid with catalytic amount of p-TsOH at $60{ }^{\circ} \mathrm{C}$ within $1 \mathrm{~h}$ in $74 \%$ yield. The compound $\mathbf{5 b}$ was partially converted to 5-ethoxy-3-(4fluorophenyl)-2 $H, 10 \mathrm{a} H$-pyrano[2,3- $b$ ]chromen-2-one 8 during chromatography on $\mathrm{SiO}_{2}$ by traces of ethanol liberated from ethyl acetate eluent. Compound $\mathbf{8}$ can be also prepared from $\mathbf{5 b}$ by its heating in $\mathrm{EtOH}$ in the presence of catalytic amount of p-TsOH (Scheme 2). 

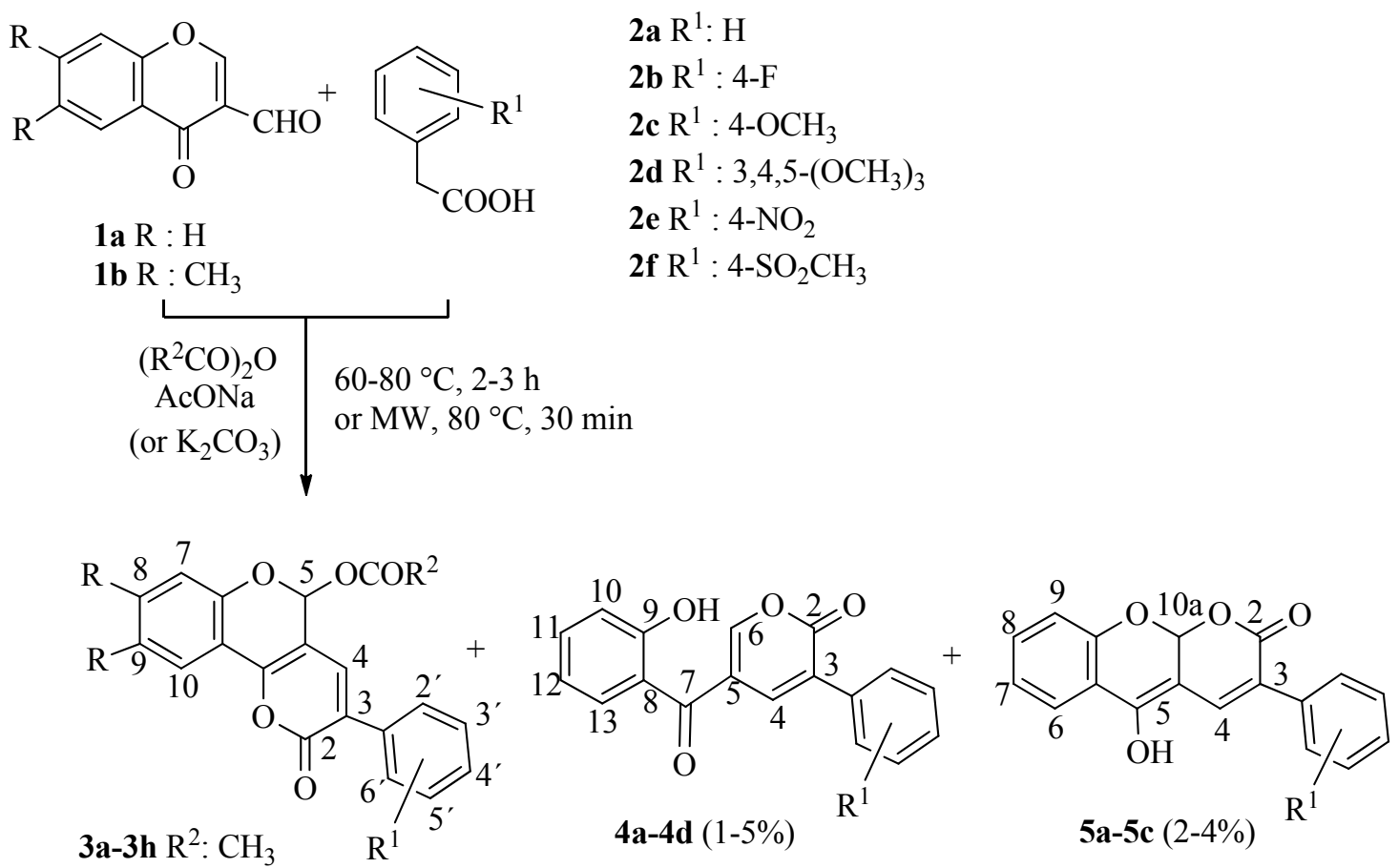

(48-85\%)

3i $\mathrm{R}^{1}: \mathrm{H}, \mathrm{R}^{2}: \mathrm{Et}$

$(80 \%)$

FLC on $\mathrm{SiO}_{2}$ EtOAc/hexane or $\mathrm{EtOH} / \mathrm{p}-\mathrm{TsOH}$



$8(5 \%)$

Scheme 1. The conditions for the synthesis of compounds $\mathbf{3 a}-\mathbf{8}$.

We have observed that the acetyloxy group at $\mathrm{C}(5)$ in the compounds 3 easily undergoes nucleophilic substitution. The acetates $\mathbf{3}$ were partially converted into their alcohols $\mathbf{6}$ during flash chromatography purification on $\mathrm{SiO}_{2}$. Therefore we have investigated the conditions for hydrolytic removing of AcO-group from 3 to prepare alcohols 6. Acid catalyzed reaction of 3a in the mixture of dioxane-water $(3: 1)$ at $50{ }^{\circ} \mathrm{C}$ within $2 \mathrm{~h}$ gave $74 \%$ of alcohol $\mathbf{6 a}$. Stirring of 3 with water in THF for $15-20 \mathrm{~h}$ at room temperature gave alcohols 6 in $26-98 \%$ yields (Scheme 2). Alkyloxy derivatives 7 were prepared by heating of $\mathbf{3}$ in alcohol (prop-2-yn-1-ol or 2-thioethanol) in the presence of catalytic amount of p-toluenesulfonic acid. The reaction heated at $60{ }^{\circ} \mathrm{C}$ for $3 \mathrm{~h}$ gave $65-75 \%$ of 7 compared to only 10 min microwave-assisted reaction that gives the yields $68-77 \%$ (Scheme 2). 


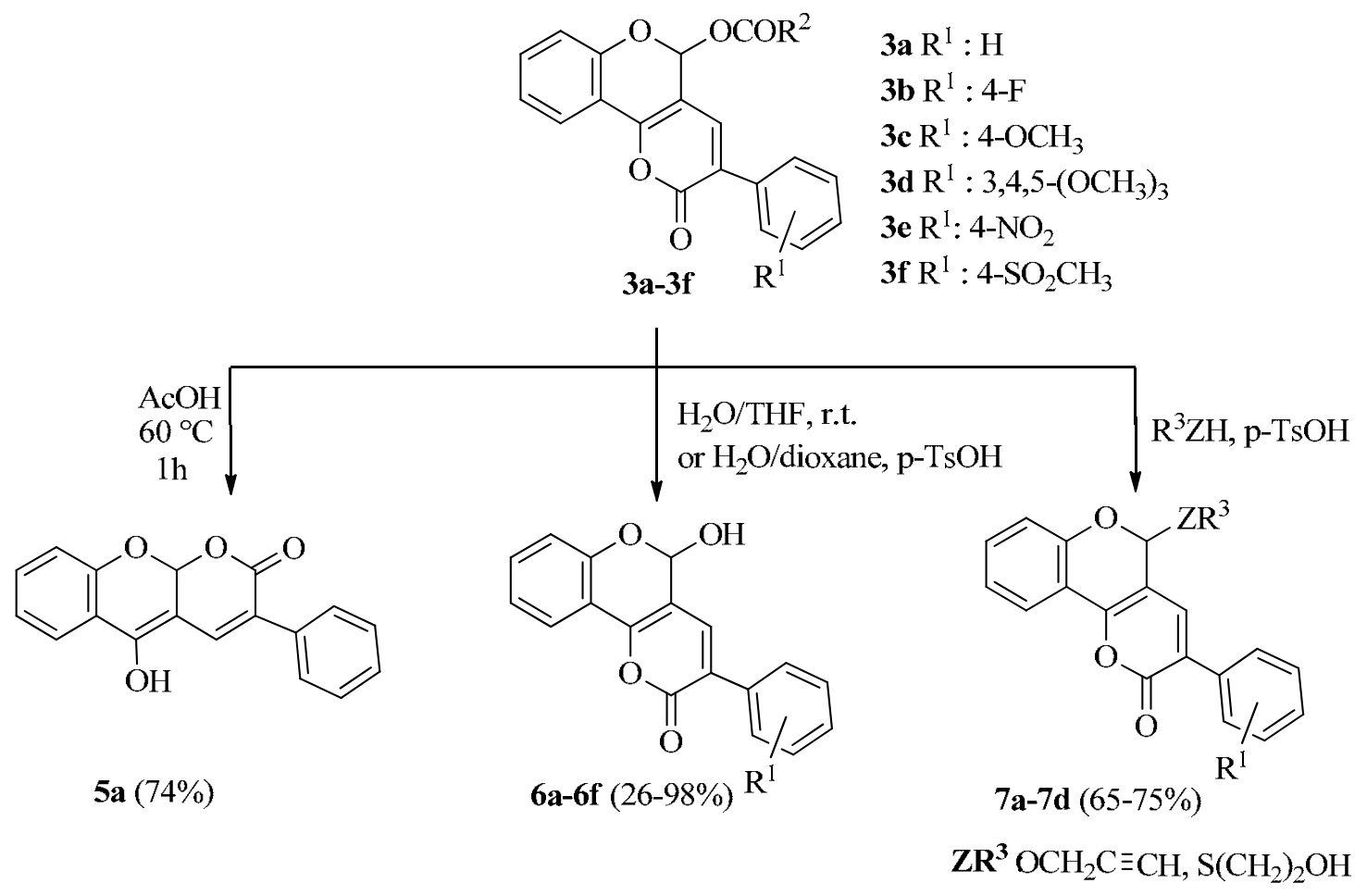

Scheme 2. The conditions for the synthesis of compounds $5 \mathbf{a}-\mathbf{7 d}$.

\section{Antineoplastic activity}

Twelve from our prepared compounds were proposed to NCI USA for biological evaluation for anticancer properties (Table 1). ${ }^{17}$

Based on structures and obtained activities we can conclude that para substitution at $\mathrm{Ph}$ $\mathrm{C}(3)$ in compounds 3 or $\mathbf{6}$ decreases antitumour properties with no relation to the electronic properties of substituents. Therefore in this case unsubstituted compounds are the most suitable $\left(\mathrm{R}^{1}: \mathrm{H}\right.$ or $\left.4-\mathrm{F}\right) \mathbf{3 g}, \mathbf{3 h}, \mathbf{3 b}$ and $\mathbf{6 a}$. Two methyl substituents in $\mathbf{3 g}, \mathbf{3 h}$ lolcated in the skeleton on $\mathrm{C}(8)$ and $\mathrm{C}(9)$ seems to be important for the increase of antitumour activity.

Table 1. The One-dose pre-screening assay results performed on 60 human tumour cell lines incubated with experimental compounds at single concentration $10^{-5} \mathrm{M}$ for $48 \mathrm{~h}$

\begin{tabular}{cccccc}
\hline Compound & $\mathrm{R}^{1}$ & $\mathrm{R}$ & $\begin{array}{c}\text { Mean } \\
\text { Growth } \\
(\%)\end{array}$ & $\begin{array}{c}\text { The Best } \\
(\%)\end{array}$ & $\begin{array}{c}\text { Further } \\
\text { evaluation }\end{array}$ \\
\hline $\mathbf{3 a}$ & $\mathrm{H}$ & $\mathrm{H}$ & $\mathrm{a}$ & & $\mathrm{N}$ \\
$\mathbf{3 b}$ & $4-\mathrm{F}$ & $\mathrm{H}$ & 78.60 & -35.40 & $60 \mathrm{CC}$ \\
$\mathbf{3 c}$ & $4-\mathrm{OMe}$ & $\mathrm{H}$ & 92.46 & -25.88 & $\mathrm{~N}$ \\
$\mathbf{3 d}$ & $3,4,5-\left(\mathrm{OCH}_{3}\right)_{3}$ & $\mathrm{H}$ & 73.73 & 53.68 & $\mathrm{~N}$ \\
$\mathbf{3 e}$ & $4-\mathrm{NO}_{2}$ & $\mathrm{H}$ & 88.96 & 20.28 & $\mathrm{~N}$ \\
$\mathbf{3 g}$ & $\mathrm{H}$ & $\mathrm{Me}$ & $\mathbf{1 0 . 9 2}$ & -42.23 & $60 \mathrm{CC}$ \\
\hline
\end{tabular}


Table 1. Continued

\begin{tabular}{|c|c|c|c|c|c|}
\hline Compound & $\mathrm{R}^{1}$ & $\mathrm{R}$ & $\begin{array}{c}\text { Mean } \\
\text { Growth } \\
(\%)\end{array}$ & $\begin{array}{c}\text { The Best } \\
(\%)\end{array}$ & $\begin{array}{c}\text { Further } \\
\text { evaluation }\end{array}$ \\
\hline $3 h$ & $4-F$ & $\mathrm{Me}$ & 59.03 & -7.18 & $60 \mathrm{CC}$ \\
\hline $6 a$ & $\mathrm{H}$ & $\mathrm{H}$ & $\mathrm{b}$ & & $60 \mathrm{CC}$ \\
\hline $6 b$ & $4-\mathrm{F}$ & $\mathrm{H}$ & 75.59 & 3.53 & $\mathrm{~N}$ \\
\hline $6 c$ & 4- $\mathrm{OCH}_{3}$ & $\mathrm{H}$ & 106.91 & 81.55 & $\mathrm{~N}$ \\
\hline $6 e$ & $4-\mathrm{NO}_{2}$ & $\mathrm{H}$ & 86.11 & -13.61 & $\mathrm{~N}$ \\
\hline $6 f$ & $4-\mathrm{SO}_{2} \mathrm{CH}_{3}$ & $\mathrm{H}$ & 91.23 & -3.93 & $\mathrm{~N}$ \\
\hline
\end{tabular}

a,b exceptionally two compounds have been pre-screened by an older method on three selected cell lines at single conc. $10^{-4} \mathrm{M}$. In this case to pass to further screenings at least one growth $\%$

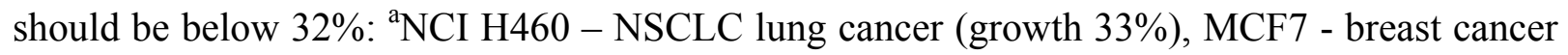
(96\%), SF-268 - CNS cancer (107\%); ${ }^{b}$ NCI H460 (2\%), MCF7 (17\%), SF-268 (25\%). Mean Growth (MID): represents average of growth \% through all 60 human tumour cell lines at initial compound concentration $10^{-5} \mathrm{M}$ compared to $100 \%$ for untreated tumour cell lines (controls). The Best inhibition activities are the border growth \% observed in individual the most sensitive human tumour cell lines at One-dose compound concentration $10^{-5} \mathrm{M}$; $\mathbf{N}$ : no further screening indicated; 60CC: means selection for screening on 60 human tumour cell line panel at five different concentrations from $10^{-4} \mathrm{M}$ to $10^{-9} \mathrm{M}$. Negative values in the table represent cytotoxic influence of screened compound.

The most promising compounds $\mathbf{3 b}, \mathbf{3 g}, \mathbf{3 h}$ and $\mathbf{6 a}$, derived from the results of One-dose prescreening, were further evaluated at five different concentrations $\left(10^{-5}\right.$ to $\left.10^{-9} \mathrm{M}\right)$ on 60 human tumour cell line panels (Table 2).

Table 2. The results of five-Dose assay performed on 60 human tumour cell lines

\begin{tabular}{ccc}
\hline Structure & \multicolumn{3}{c}{$\begin{array}{c}\mathrm{GI}_{50}\left[10^{-6} \mathrm{M}\right] \\
\text { Mean growth inhibition } 60 \mathrm{CC} \text { and the best individual cell } \\
\text { lines values }\end{array}$} \\
\hline $\mathbf{3 b}$ & 22.4 & 2.88 \\
$\mathbf{3 g}$ & $\mathbf{0 . 4 4 7}$ & $\mathbf{0 . 0 3 1}$ \\
$\mathbf{3 h}$ & 5.37 & 1.20 \\
$\mathbf{6 a}$ & 7.41 & 1.62 \\
\hline
\end{tabular}

Compound 3g was shown to be the most active in slowing down tumour cell division compare to the control. Its structure contains unsubstituted phenyl group at $\mathrm{C}(3)$ and two methyl substituents at $\mathrm{C}(8)$ and $\mathrm{C}(9)$ (Table 1). This compound possesses submicromolar mean growth 
inhibition activity over all 60 cancer cell lines panel $\left(\mathrm{GI}_{50}: 447 \mathrm{nM}\right)$. Individual tumour cell lines (e.g. Leukemia, NSCLC, Melanoma) have shown even nanomolar sensitivities for this compound $\left(\mathrm{GI}_{50}: 31\right.$ - $63 \mathrm{nM}$, not stated in this paper).

The exact mechanism of antitumour activity of compound $\mathbf{3 g}$ is not known. We believe that 3g interacts with tubulines. The proposed mechanism is based on comparison of $\mathrm{GI}_{50}$ activity profile for individual tumour cell lines (kind of finger print) with profile of standard antitumour compounds having known antitumour mechanism. Compare analysis software is accessible online and it is free of charge. ${ }^{18}$ The compare analysis has been done with $\mathbf{3 g}$ inhibitor experimental $\mathrm{GI}_{50}$ values determined for all 60 human tumour cell lines (CC60 assays). These data were compared to sets of $\mathrm{GI}_{50}$ values of STANDARD_AGENTS taken from NCI database. A correlation between our most active compound 3g (NSC:745523) with compounds possessing typical antitubuline interactions has been obtained: e.g. rhizoxin with NCI code NSC:S332598 (correlation coefficient, 0.597), maytansine NSC:S153858 (0.438), paclitaxel NSC:S125973 (0.400), S-trityl-L-cysteine NSC:S83265 (0.374), vinblastine sulfate NSC:S49842 (0.358). The list of compounds with known antitubuline mechanism is published in NCI databases. ${ }^{19}$

\section{Conclusions}

A new leading skeleton with 3-phenyl-2H,5H-pyrano[3,2-c]chromen-2-one structure has been identified thank to the NCI tumour cell lines screening possibilities. 3-Phenyl-2-oxo- $2 \mathrm{H}, 5 \mathrm{H}$ pyrano[3,2-c]chromen-5-yl acetates 3 were synthesized by the reaction of 4-oxo-4H-chromen-3carbaldehydes 1 and substituted phenylacetic acids $\mathbf{2}$ in acetic anhydride. At stronger conditions, small amounts of two by-products 5-(2-hydroxybenzoyl)-3-phenyl-2H-pyran-2-ones 4 and 5hydroxy- $2 H, 10 \mathrm{a} H$-pyrano[2,3-b]chromen-2-ones $\mathbf{5}$ were also obtained. Reaction of $\mathbf{3}$ with alcohols or water gave corresponding 5-hydroxy- and 5-alkyloxy $2 \mathrm{H}, 5 \mathrm{H}$-pyrano[3,2-c]chromen2-ones 6 and 7, respectively. Synthesis of some derivatives 3 and 7 were significantly accelerated under microwave irradiation and completed within 10-30 min. Based on the antitumour screening results and Beilstein database searches we found that 3-phenyl-2H,5H-pyrano[3,2c]chromen-2-ones represent a new type of antitumour compounds suitable for further development. $^{20}$ In our case the most active compounds were $\mathbf{3 g}, \mathbf{3 h}, \mathbf{3 b}$ and $\mathbf{6 a}\left(\mathrm{R}^{1}\right.$ : H or $\left.4-\mathrm{F}\right)$. Para substituents $\mathrm{R}^{1}$ at $\mathrm{Ph}-\mathrm{C}(3)$ in compounds $\mathbf{3}$ or $\mathbf{6}$ decreasing antitumour activity independently on their electronic properties. On the other site methyl groups at $\mathrm{C}(8)$ and $\mathrm{C}(9)$ in 3g, 3h seems to be important for observed activities. We proposed that the antitumour activity of $\mathbf{3 g}$ performs via interaction with tubulines. This was based on comparisons of $\mathbf{3 g} \mathrm{GI}_{50}$ profile with similar $\mathrm{GI}_{50} \mathrm{CC} 60$ profiles of the standard agents from NCI database. 


\section{Experimental Section}

General. Melting points were determined on a Kofler hot plate apparatus and are uncorrected. ${ }^{1} \mathrm{H}$ NMR/ ${ }^{13} \mathrm{C}$ NMR spectra were obtained on a $300 \mathrm{MHz} / 75 \mathrm{MHz}$ VARIAN GEMINI 2000 spectrometer in $\mathrm{CDCl}_{3}$ or DMSO- $d_{6}$ with tetramethylsilane as an internal standard. Elemental analyses were performed on CARLO ERBA STRUMENTAZIONE 1106 apparatus. IR spectra were recorded on FTIR-ATR REACT IR 1000 spectrometer in $\mathrm{KBr}$ or nujol. All microwaveassisted reactions were performed in an Initiator BIOTAGE microwave synthesizer. All solvents were distilled and dried appropriately prior to use. The course of reactions was monitored by TLC in ethyl acetate - hexane. Flash column chromatography was performed at $\mathrm{SiO}_{2}(\mathrm{Merck}$ Silica gel 60) with hexane/ethyl acetate as eluent. 4-Oxo-4H-chromene-3-carbaldehyde was synthesized according to the method, described by Nohara. ${ }^{21}$ The other chemicals were purchased from the suppliers as the highest purity grade. Antitumour activities were determined via Developmental Therapeutics Program (DTP) NCI / NIH in USA. ${ }^{22}$

\section{General procedures for ( $\mathrm{R}^{1}$-phenyl)-2-oxo-2H,5H-pyrano[3,2-c]chromen-5-yl acetates (3a-} 3h)

Method A. A mixture of 4-oxo-4H-chromene-3-carbaldehyde 1a or 1b $(20.0 \mathrm{mmol}, 1.00 \mathrm{~mol}$ eq) and substituted phenylacetic acid $2(20.0 \mathrm{mmol}, 1.00 \mathrm{~mol} \mathrm{eq})$ in freshly distilled acetic anhydride $(10 \mathrm{~mL})$ and fused sodium acetate $(45.0 \mathrm{mg}, 1.14 \mathrm{mmol}, 0.06 \mathrm{~mol} \mathrm{eq})$ was stirred for 2 $\mathrm{h}$ at $60-70^{\circ} \mathrm{C}$. After cooling the solid product 3 was filtered off, washed with cold diethyl ether and crystallized from dioxane abs.

Method B. A mixture of 4-oxo-4H-chromene-3-carbaldehyde 1a (20.0 mmol, $1.00 \mathrm{~mol}$ eq) and substituted phenylacetic acid $2(20.0 \mathrm{mmol}, 1.00 \mathrm{~mol}$ eq) in freshly distilled acetic anhydride (10 $\mathrm{mL})$ and potassium carbonate $(10.0 \mathrm{mg}, 0.72 \mathrm{mmol}, 0.04 \mathrm{~mol} \mathrm{eq})$ was irradiated in microwave oven at $80{ }^{\circ} \mathrm{C}$ and $20 \mathrm{~W}$ for $30 \mathrm{~min}$. The product 3 was isolated and purified in the same manner as described in Method A.

Method C. A mixture of 4-oxo-4H-chromene-3-carbaldehyde 1a (500.0 mg, 2.87 mmol, 1.00 mol eq) and substituted phenylacetic acid 2 (3.14 mmol, $1.09 \mathrm{~mol} \mathrm{eq})$ in freshly distilled acetic anhydride $(1.5 \mathrm{~mL})$ and fused sodium acetate $(49.4 \mathrm{mg}, 0.21 \mathrm{mmol}, 0.07 \mathrm{~mol} \mathrm{eq})$ was stirred for $12-16 \mathrm{~h}$ at $100{ }^{\circ} \mathrm{C}$ under argon atmosphere. After cooling the yellow crystals were filtered off, washed with ethyl acetate $(2 \mathrm{~mL})$ and re-crystallized from ethyl acetate to give products $\mathbf{3}$. After evaporation of solvents, the products $\mathbf{4}$ and $\mathbf{5}$, respectively were separated by flash chromatography on $\mathrm{SiO}_{2}$ with hexane/ethyl acetate $(3: 1)$.

3-Phenyl-2-oxo-2H,5H-pyrano[3,2-c]chromen-5-yl acetate (3a). Method A, work-up as described above gave $81 \%$ of $\mathbf{3 a}$, (method B, 85\%, method C, 11\%) as yellow powder: mp 230$232{ }^{\circ} \mathrm{C}$ (ethyl acetate). Anal. calcd. for $\mathrm{C}_{20} \mathrm{H}_{14} \mathrm{O}_{5}$ (334.3) requires: C, 71.85; H, 4.22. Found: C, 71.67; H, 4.24. IR v(KBr): 1717, 1651, 1638, 1565, 1550, 1490, 1460, 1376, 1222, 1198 , 1116, 1043, 1001, 949, 893, 783, $760 \mathrm{~cm}^{-1}$; ${ }^{1} \mathrm{H}$ NMR (300 MHz, DMSO-d $) \delta 2.00\left(3 \mathrm{H}, \mathrm{s}, \mathrm{CH}_{3}\right)$, $7.13(1 \mathrm{H}, \mathrm{d}, J=8.2,0.8 \mathrm{~Hz}, \mathrm{H}-7), 7.21(1 \mathrm{H}, \mathrm{ddd}, J=7.7,7.1,0.8 \mathrm{~Hz}, \mathrm{H}-9), 7.39$ (1H, s, H-5), 
7.41 -7.45 (3H, m, H-3',4',5'), 7.48 (1H, ddd, $J=7.7,7.0,1.4 \mathrm{~Hz}, \mathrm{H}-8), 7.51$ (1H, s, H-4), 7.68-

$7.71\left(2 \mathrm{H}, \mathrm{m}, \mathrm{H}-2^{\prime}, 6^{\prime}\right), 7.92(1 \mathrm{H}, \mathrm{dd}, J=7.7,1.4 \mathrm{~Hz}, \mathrm{H}-10) ;{ }^{13} \mathrm{C}$ NMR $\left(75 \mathrm{MHz}, \mathrm{DMSO}-d_{6}\right) \delta$ $21.1,91.3,109.8,114.5,117.6,122.1,122.2,124.7,128.1,128.1,128.4,128.4,128.5,132.5$, $134.5,139.9,150.1,152.7,159.8,172.1$.

3-(4-Fluorophenyl)-2-oxo-2H,5H-pyrano[3,2-c]chromen-5-yl acetate (3b). Method A, workup as described above gave $60 \%$ of $\mathbf{3 b}(\operatorname{method} \mathrm{B}, 70 \%$, method $\mathrm{C}, 40 \%)$ as yellow powder: $\mathrm{mp}$ 244-246 ${ }^{\circ} \mathrm{C}$ (ethyl acetate). Anal. calcd. for $\mathrm{C}_{20} \mathrm{H}_{13} \mathrm{FO}_{5}$ (352.3): C, 68.18; H, 3.72. Found: $\mathrm{C}, 68.41 ; \mathrm{H}, 3.59$. IR $v(\mathrm{KBr}): 1721,1644,1601,1559,1509,1459,1370,1269,1235,1154$, $1038,937,837,760 \mathrm{~cm}^{-1} .{ }^{1} \mathrm{H}$ NMR $\left(300 \mathrm{MHz}, \mathrm{DMSO}-d_{6}\right) \delta 2.02\left(\mathrm{~s}, 3 \mathrm{H}, \mathrm{CH}_{3}\right), 7.21(\mathrm{dd}, 1 \mathrm{H}, J=$ 8.3, 0.7 Hz, H-7), $7.25-7.36$ (m, 3H, H-9,3',5'), 7.45 (s, 1H, H-5), 7.54 (ddd, 1H, J = 8.3, 7.5, 1.6 $\mathrm{Hz}, \mathrm{H}-8), 7.77-7.84$ (m, 3H, H-10,2',6'), 8.00 (s, 1H, H-4). ${ }^{13} \mathrm{C}$ NMR (75 MHz, DMSO- $\left.d_{6}\right) \delta$ 20.7, 89.2, 106.0, 114.0, 115.2, 117.4, 117.4, 122.4, 123.4, 123.9, 130.2, 130.6, 133.0, 139.2, $150.9,151.6,151.6,159.3,162.1,169.0$.

3-(4-Methoxyphenyl)-2-oxo-2H,5H-pyrano[3,2-c]chromen-5-yl acetate (3c). Method B, work-up as described above gave $68 \%$ of 3a (method C, 54\%) as yellow powder: mp 226-227 ${ }^{\circ} \mathrm{C}$ (ethyl acetate). Anal. calcd. for $\mathrm{C}_{21} \mathrm{H}_{16} \mathrm{O}_{6}$ (364.4): $\mathrm{C}, 69.23$; H, 4.43. Found: $\mathrm{C}, 69.61$; $\mathrm{H}, 4.59$. IR $v(\mathrm{KBr}): 1713,1648,1609,1559,1513,1459,1250,1177,1030,941,826,760 \mathrm{~cm}^{-1}$. ${ }^{1} \mathrm{H}-\mathrm{NMR}\left(300 \mathrm{MHz}, \mathrm{DMSO}-d_{6}\right) \delta 2.01\left(\mathrm{~s}, 3 \mathrm{H}, \mathrm{CH}_{3}\right), 3.80\left(\mathrm{~s}, 3 \mathrm{H}, \mathrm{CH}_{3} \mathrm{O}\right), 6.99-7.06(\mathrm{~m}, 2 \mathrm{H}, \mathrm{H}-$ 3',5'), 7.19 (dd, 1H, $J=8.2,2.4 \mathrm{~Hz}, \mathrm{H}-7), 7.27$ (ddd, 1H, $J=8.0,7.8,2.4 \mathrm{~Hz}, \mathrm{H}-9), 7.44$ (s, 1H, H-5), 7.52 (ddd, $1 \mathrm{H}, J=8.2,7.8,1.5 \mathrm{~Hz}, \mathrm{H}-8), 7.68-7.75$ (m, 2H, H-2',6'), 7.78 (dd, 1H, $J=8.0$, $1.5 \mathrm{~Hz}, \mathrm{H}-10), 7.92$ (s, $1 \mathrm{H}, \mathrm{H}-4) .{ }^{13} \mathrm{C}$ NMR (75 MHz, DMSO-d $\left.d_{6}\right) \delta 20.8,55.2,89.3,106.1,113.8$, $113.8,114.2$, 117.4, 122.3, 123.4, 124.7, 126.4, 129.4, 129.4, 132.7, 137.7, 150.1, 151.4, 159.5, 159.6, 169.1 .

3-(3,4,5-Trimethoxyphenyl)-2-oxo-2H,5H-pyrano[3,2-c]chromen-5-yl acetate (3d). Method A, work-up as described above gave $53 \%$ of 3d (method C, 16\%) as yellow powder: mp 175$180{ }^{\circ} \mathrm{C}$ (ethyl acetate). Anal. calcd. for $\mathrm{C}_{23} \mathrm{H}_{20} \mathrm{O}_{8}$ (424.4): $\mathrm{C}, 65.09 ; \mathrm{H}, 4.75$. Found: $\mathrm{C}, 65.33 ; \mathrm{H}$, 4.44. IR $v(\mathrm{KBr}): 1733,1648,1555,1462,1374,1331,1239,1192,1127,1003,926,764 \mathrm{~cm}^{-1}$. ${ }^{1} \mathrm{H}$ NMR (300 MHz, DMSO-d $\left.)\right) \delta 2.02\left(\mathrm{~s}, 3 \mathrm{H}, \mathrm{CH}_{3}\right), 3.71\left(\mathrm{~s}, 3 \mathrm{H}, \mathrm{CH}_{3} \mathrm{O}\right), 3.83(\mathrm{~s}, 6 \mathrm{H}, 2 \mathrm{x}$ $\mathrm{CH}_{3} \mathrm{O}$ ), 7.08 (s, 2H, H-2',6'), 7.21 (dd, 1H, J=8.1, 1.0, H-7), 7.29 (ddd, 1H, $J=7.7,7.3,1.0 \mathrm{~Hz}$, H-9), 7.44 (s, 1H, H-5), 7.54 (ddd, 1H, $J=8.1,7.3,1.3 \mathrm{~Hz}, \mathrm{H}-8), 7.80$ (dd, $1 \mathrm{H}, J=7.7,1.3 \mathrm{~Hz}$, $\mathrm{H}-10), 8.02$ (s, 1H, H-4). ${ }^{13} \mathrm{C}$ NMR (75 MHz, DMSO- $\left.d_{6}\right) \delta 20.7,55.9,60.0,89.1,105.7,105.8$, $114.0,117.3,122.3,123.4,124.7,129.5,132.9,138.0,138.8,150.5,151.4,152.5,159.1,169.1$.

3-(4-Nitrophenyl)-2-oxo-2H,5H-pyrano[3,2-c]chromen-5-yl acetate (3e). Method A, work-up as described above gave $49 \%$ of $\mathbf{3 e}\left(\operatorname{method} \mathrm{B}, 72 \%\right.$ ) as yellow powder: mp $249-251{ }^{\circ} \mathrm{C}$ (ethyl acetate). Anal. calcd. for $\mathrm{C}_{20} \mathrm{H}_{13} \mathrm{NO}_{7}$ (379.3): C, 63.33; H, 3.45; N, 3.69. Found: C, 62.86; H, 3.90; N, 3.75. IR $v$ (nujol): 1763, 1734, 1639, 1601, 1547, 1517, 1346, 1216, 1139, 1110, 1042, 1000, 938, 852, 767, 738, 691, 621. ${ }^{1} \mathrm{H}$ NMR (300 MHz, DMSO- $d_{6}$, $\delta 2.02\left(\mathrm{~s}, 3 \mathrm{H}, \mathrm{CH}_{3} \mathrm{O}\right), 7.22$ (dd, $1 \mathrm{H}, J=8.3,1.0 \mathrm{~Hz}, \mathrm{H}-7), 7.30$ (ddd, 1H, $J=7.9,7.5,1.0 \mathrm{~Hz}, \mathrm{H}-9), 7.46$ (s, 1H, H-5), 7.57 (ddd, $1 \mathrm{H}, J=8.3,7.5,1.6 \mathrm{~Hz}, \mathrm{H}-8), 7.83$ (dd, 1H, $J=7.9,1.6 \mathrm{~Hz}, \mathrm{H}-10), 8.02-8.08$ (m, 2H, H$\left.2^{\prime}, 6^{\prime}\right), 8.23$ (s, 1H, H-4), 8.30-8.36 (m, 2H, H-3',5'). ${ }^{13} \mathrm{C}$ NMR (75 MHz, DMSO-d $)$ ) $\delta 20.8,89.1$, 
$106.0,113.8,117.5,122.5,122.8,123.5,123.4,129.1,133.6,140.8,141.5,146.9,151.9,152.3$, $158.9,169.1$.

3-[(4-Methylsulfonyl)phenyl]-2-oxo-2H,5H-pyrano[3,2-c]chromen-5-yl acetate (3f). Method A, work-up as described above gave $70 \%$ of $\mathbf{3 f}$ as yellow powder: mp $240-242{ }^{\circ} \mathrm{C}$ (ethyl acetate). Anal. calcd. for $\mathrm{C}_{21} \mathrm{H}_{18} \mathrm{O}_{7} \mathrm{~S}$ (412.4): C, 61.16; H, 3.91; S, 7.77. Found: C, 61.05; H, 4.36; S, 7.54. IR $v$ (nujol): $1755,1733,1646,1556,1292,1219,1194,1148,1044,999,925,846,765 \mathrm{~cm}^{-1} .{ }^{1} \mathrm{H}$ NMR (300 MHz, DMSO- $\left.d_{6}\right) \delta 2.02\left(\mathrm{~s}, 3 \mathrm{H}, \mathrm{CH}_{3} \mathrm{O}\right), 7.22(\mathrm{dd}, 1 \mathrm{H}, J=8.2,0.8 \mathrm{~Hz}, \mathrm{H}-7), 7.30$ (ddd, 1H, $J=7.9,0.8 \mathrm{~Hz}, \mathrm{H}-9$ ), 7.48 (s, 1H, H-5), 7.57 (ddd, 1H, J= 8.2, 7.3, $1.4 \mathrm{~Hz}, \mathrm{H}-8$ ), 7.83 $(\mathrm{dd}, 1 \mathrm{H}, J=7.9,1.4 \mathrm{~Hz}, \mathrm{H}-10), 8.00-8.04$ (m, 4H, H-2', 3', 5',6'), 8.02 (s, 1H, H-4). ${ }^{13} \mathrm{C}$ NMR (75 $\left.\mathrm{MHz}, \mathrm{DMSO}-d_{6}\right) \delta 21.5,43.9,91.6,110.2,114.7,118.1,122.6,122.8,123.3,127.4,129.3$, $133.3,139.9,140.6,141.9,151.6,153.4,159.9,172.5$.

8,9-Dimethyl-2-oxo-3-phenyl-2H,5H-pyrano[3,2-c]chromen-5-yl acetate (3g). Method A, work-up as described above gave $74 \%$ of $\mathbf{3 g}$ as yellow powder: mp $205-207{ }^{\circ} \mathrm{C}$ (ethyl acetate). Anal. calcd. for $\mathrm{C}_{22} \mathrm{H}_{18} \mathrm{O}_{5}$ (362.4): C, 72.92; H, 5.01. Found: C, 73.09; H, 4.97. IR v (KBr): 3541, 3034, 2917, 1725, 1714, 1646, 1551, 1502, 1357, 1227, 1187, 1136, 1024, 1001, 935, 912, 887, 783, 699, $521 \mathrm{~cm}^{-1} .{ }^{1} \mathrm{H}$ NMR (300 MHz, DMSO-d $): 1.97\left(3 \mathrm{H}, \mathrm{s}, \mathrm{CH}_{3}\right), 2.27\left(6 \mathrm{H}, \mathrm{s}, 2 \mathrm{x} \mathrm{CH}_{3}\right)$, 7.01 (1H, s, H-7), 7.39 (1H, s, H-5), 7.40-7.48 (3H, m, H-3', 4', 5'), 7.54 (1H, s, H-4), 7.70-7.74 $\left(2 \mathrm{H}, \mathrm{m}, \mathrm{H}-2^{\prime}, 6^{\prime}\right), 7.92(1 \mathrm{H}, \mathrm{s}, \mathrm{H}-10) ;{ }^{13} \mathrm{C}$ NMR (75 MHz, DMSO- $\left.d_{6}\right): 18.6,19.8,20.7,89.5$, $105.3,109.0,111.5,118.1,122.5,123.9,127.9,128.0,128.3,128.5,131.8,134.4,139.6,142.8$, $149.9,151.5,159.4,169.1$.

\section{3-(4-Fluorophenyl)-8,9-dimethyl-2-oxo-2H,5H-pyrano[3,2-c]chromen-5-yl acetate (3h).}

Method A, work-up as described above gave $73 \%$ of $\mathbf{3 h}$ as yellow powder: mp $246-248{ }^{\circ} \mathrm{C}$ (ethyl acetate). Anal. calcd. for $\mathrm{C}_{22} \mathrm{H}_{17} \mathrm{FO}_{5}$ (380.4): C, 69.47; H, 4.50. Found: C, 69.29; H, 4.47. IR $v(\mathrm{KBr}): 3443,2926,2857,1728,1686,1638,1547,1510,1459,1377,1357,1303,1233$, 1163, 1105, 1051, 1030, 965, 893, 837, 774, 663, $527 \mathrm{~cm}^{-1}$. ${ }^{1} \mathrm{H}$ NMR (300 MHz, DMSO-d6): $2.00\left(3 \mathrm{H}, \mathrm{s}, \mathrm{CH}_{3}\right), 2.25\left(3 \mathrm{H}, \mathrm{s}, \mathrm{CH}_{3}\right), 2.48\left(3 \mathrm{H}, \mathrm{s}, \mathrm{CH}_{3}\right), 7.01(1 \mathrm{H}, \mathrm{s}, \mathrm{H}-5), 7.29(2 \mathrm{H}, \mathrm{ddd}, J=$ 8.9, 7.4, 2.1 Hz, H-2', 6'), 7.37 (1H, s, H-7), 7.54 (1H, s, H-10), 7.77 (2H, ddd, J = 8.9, 7.7, 2.0 Hz, H-3', 5'), 7.95 (1H, s, H-4); ${ }^{13} \mathrm{C}$ NMR (75 MHz, DMSO-d6): 18.6, 19.8, 20.8, 91.2, 109.0, $111.9,115.1,115.3,118.3,122.3,127.4,130.1,130.2,131.9,133.6,134.9,140.0,142.0,150.7$, $150.9,159.9,161.6$.

General procedures for 3-(4-fluorophenyl)-2-oxo-2H,5H-pyrano[3,2-c]chromen-5-yl propionate (3i)

Method D. A mixture of 4-oxo-4H-chromene-3-carbaldehyde 1 (3.00 mmol, $1.00 \mathrm{~mol} \mathrm{eq})$ and 4fluorophenylacetic acid $\mathbf{2 b}(3.00 \mathrm{mmol}, 1.00 \mathrm{~mol}$ eq) in propionic anhydride $(5 \mathrm{~mL})$ and potassium carbonate $(50.0 \mathrm{mg}, 0.36 \mathrm{mmol}, 0.12 \mathrm{~mol} \mathrm{eq})$ was stirred for $2 \mathrm{~h}$ at $80{ }^{\circ} \mathrm{C}$. After cooling, diethyl ether $(20 \mathrm{~mL})$ was added and the mixture was cooled at $-10{ }^{\circ} \mathrm{C}$ for $1 \mathrm{~h}$. The solid product was filtered off, washed with cold diethyl ether and crystallized from dioxane to give $80 \%$ of $\mathbf{3 i}$ as yellow powder. 
Method E. A mixture of 4-oxo-4H-chromene-3-carbaldehyde 1 (1.00 mmol, $1.00 \mathrm{~mol}$ eq) and 4fluorophenylacetic acid $\mathbf{2 b}(1.00 \mathrm{mmol}, 1.00 \mathrm{~mol}$ eq) in propionic anhydride $(2 \mathrm{~mL})$ and potassium carbonate $(10.0 \mathrm{mg}, 0.07 \mathrm{mmol}, 0.002 \mathrm{~mol} \mathrm{eq})$ was irradiated in microwave oven at $110{ }^{\circ} \mathrm{C}$ and $20 \mathrm{~W}$ for $30 \mathrm{~min}$. The product $3 \mathbf{i}$ was isolated and purified in the same manner as described in Method A to give $82 \%$ of $3 \mathbf{i}$ as yellow powder.

Method D, work-up as described above gave $80 \%$ of $\mathbf{3 i}$ (method E, 82\%) as yellow powder, m.p. 255-257 ${ }^{\circ} \mathrm{C}$ (ethyl acetate). Anal. calcd. for $\mathrm{C}_{21} \mathrm{H}_{15} \mathrm{FO}_{5}$ (366.3): C, 69.85; H, 4.13. Found: $\mathrm{C}$, 70.08; H, 4.16. IR $v$ (nujol): 1748, 1730, 1645, 1557, 1292, 1220, 1195, 1152, 1045, 999, 930, $845,770 \mathrm{~cm}^{-1} .{ }^{1} \mathrm{H}$ NMR $\left(300 \mathrm{MHz}, \mathrm{DMSO}-d_{6}\right) \delta 0.99\left(\mathrm{t}, 3 \mathrm{H}, J=7.4 \mathrm{~Hz}, \mathrm{CH}_{3}\right), 2.30$ (q, $2 \mathrm{H}, J=$ $7.4 \mathrm{~Hz}, \mathrm{CH}_{2}$ ), 7.20 (d, 1H, J=8.2 Hz, H-7), $7.26-7.35$ (m, 3H, H-9,3',5'), 7.47 (s, 1H, H-5), 7.54 (ddd, 1H, $J=8.2,7.1,1.6 \mathrm{~Hz}, \mathrm{H}-8), 7.77-7.83$ (m, 3H, H-10,2',6'), 8.01 (s, 1H, H-4).

\section{General procedure for 5-(2-hydroxybenzoyl)-3-( $\mathbf{R}^{\mathbf{1}}$-phenyl)-2H-pyran-2-ones (4a-4d)}

Compounds 4 were isolated by flash chromatography from the mother liqueur obtained from the reaction after removing product 3 by filtration. (see Method $\mathrm{C}$ ).

5-(2-Hydroxybenzoyl)-3-phenyl-2H-pyran-2-one (4a). Method $\mathrm{C}$, work-up as described above gave $5 \%$ of $4 \mathbf{a}$, as yellow powder: $\mathrm{mp} 270-273{ }^{\circ} \mathrm{C} . \mathrm{R}_{\mathrm{f}}=0.24$ in EtOAc/hexane 1:1. Anal. calcd. for $\mathrm{C}_{18} \mathrm{H}_{12} \mathrm{O}_{4}$ (292.3): C, 73.97; H, 4.14. Found: 73.74; H, 4.22. IR $v(\mathrm{KBr}): 1650,1575,1470$, $1412,1356,1290,1228,1125,1007,909,756 \mathrm{~cm}^{-1} .{ }^{1} \mathrm{H}$ NMR (300 MHz, DMSO-d $\left.d_{6}\right) \delta 7.06-7.33$ (m, 7H, H-4,6,Ph), 7.47 (ddd, 1H, $J=7.9,7.0,1.1 \mathrm{~Hz}, \mathrm{H}-12), 7.51$ (d, 1H, $J=8.6 \mathrm{~Hz}, \mathrm{H}-10$ ), 7.75 (ddd, 1H, $J=8.6,7.0,1.7 \mathrm{~Hz}, \mathrm{H}-11), 8.08$ (dd, 1H, $J=7.9,1.7 \mathrm{~Hz}, \mathrm{H}-13$ ), OH not seen.

3-(4-Fluorophenyl)-5-(2-hydroxybenzoyl)-2H-pyran-2-one (4b). Method $\mathrm{C}$, work-up as described above gave $2 \%$ of $\mathbf{4 b}$, as yellow powder: $\mathrm{mp} 290-292{ }^{\circ} \mathrm{C}$. $\mathrm{R}_{\mathrm{f}}=0.22$ in EtOAc/hexane 1:3. Anal. calcd. for $\mathrm{C}_{18} \mathrm{H}_{11} \mathrm{FO}_{4}$ (310.1): C, 69.68; H, 3.57. Found: C, 69.18; H, 3.47. IR v (KBr): 1721, 1509, 1462, 1262, 1223, 1119, 1073, 1019, 799, 744, $694 \mathrm{~cm}^{-1} .{ }^{1} \mathrm{H}$ NMR (300 MHz, DMSO- $\left.d_{6}\right) \delta$ 7.17-7.25 (m, 2H, H-3',5'), 7.25-7.32 (m, 2H, H-2',6'), 7.51 (ddd, $1 \mathrm{H}, J=8.0,7.1$, $1.0 \mathrm{~Hz}, \mathrm{H}-12), 7.57$ (d, 1H, J= 8.5, 1.0, H-10), 7.60 (d, 1H, J=0.9, H-6), 7.77 (d, 1H, J=0.9, H4), 7.81 (ddd, $1 \mathrm{H}, J=8.5,7.1,1.7 \mathrm{~Hz}, \mathrm{H}-11$ ), 8.09 (dd, 1H, $J=8.0,1.7, \mathrm{H}-13$ ), 12.89 (br s, $1 \mathrm{H}$, $\mathrm{OH}) .{ }^{13} \mathrm{C}$ NMR $\left(300 \mathrm{MHz}, \mathrm{DMSO}-d_{6}\right) \delta 115.5,118.5,119.0,123.0,125.3,126.0,130.1,131.6$, $131.8,134.0,134.6,155.2,156.4,161.6,167.5,175.1$.

5-(2-Hydroxybenzoyl)-3-(4-methoxyphenyl)-2H-pyran-2-one (4c). Method $\mathrm{C}$, work-up as described above gave $1 \%$ of $\mathbf{4 c}$, as yellow powder: $\mathrm{mp} 235-238^{\circ} \mathrm{C} . \mathrm{R}_{\mathrm{f}}=0.19$ in EtOAc/hexane 1:1. Anal. calcd. for $\mathrm{C}_{19} \mathrm{H}_{14} \mathrm{O}_{5}$ (322.3): C, 70.80; H, 4.38. Found: C, 70.53; H, 4.64. IR $v(\mathrm{KBr})$ : $1725,1515,1468,1274,1230,1121,1081,1025,799,748 \mathrm{~cm}^{-1} .{ }^{1} \mathrm{H}$ NMR $\left(300 \mathrm{MHz}, \mathrm{DMSO}-d_{6}\right)$ $\delta 3.77\left(\mathrm{~s}, 1 \mathrm{H}, \mathrm{CH}_{3} \mathrm{O}\right), 6.92-6.98\left(\mathrm{~m}, 2 \mathrm{H}, \mathrm{H}-3^{\prime}, 5^{\prime}\right), 7.12-7.17\left(\mathrm{~m}, 2 \mathrm{H}, \mathrm{H}-2^{\prime}, 6^{\prime}\right), 7.51$ (ddd, $1 \mathrm{H}, J=$ 8.0, 7.1, $1.0 \mathrm{~Hz}, \mathrm{H}-12), 7.55$ (d, 1H, $J=0.9, \mathrm{H}-6), 7.57$ (d, 1H, $J=8.5 \mathrm{~Hz}, \mathrm{H}-10), 7.72$ (d, 1H, $J$ $=0.9 \mathrm{~Hz}, \mathrm{H}-4), 7.81(\mathrm{ddd}, 1 \mathrm{H}, J=8.5,7.1,1.7 \mathrm{~Hz}, \mathrm{H}-11), 8.09$ (dd, $1 \mathrm{H}, J=8.0,1.7 \mathrm{~Hz}, \mathrm{H}-13)$, 12.73 (br s, $1 \mathrm{H}, \mathrm{OH}) .{ }^{13} \mathrm{C}$ NMR (300 MHz, DMSO-d $) \delta 54.9,113.9,118.4,119.1,122.9,125.2$, $125.9,127.4,129.0,130.2,130.5,134.5,155.0,156.2,158.6,167.8,175.1$. 
5-(2-Hydroxybenzoyl)-3-(3,4,5-trimethoxyphenyl)-2H-pyran-2-one (4d). Method C, work-up as described above gave $5 \%$ of $\mathbf{4 d}$, as yellow powder: $\mathrm{mp} 230-233{ }^{\circ} \mathrm{C} . \mathrm{R}_{\mathrm{f}}=0.26$ in EtOAc/hexane 1:2. Anal. calcd. for $\mathrm{C}_{21} \mathrm{H}_{18} \mathrm{O}_{7}$ (322.1): C, 70.80; H, 4.38. Found: C, 70.35; $\mathrm{H}$, 4.51. IR $v(\mathrm{KBr}): 1655,1578,1466,1412,1354,1285,1227,1123,1003,903,756 \mathrm{~cm}^{-1} .{ }^{1} \mathrm{H}$ NMR (300 MHz, $\left.\mathrm{CDCl}_{3}\right) \delta 3.80\left(\mathrm{~s}, 6 \mathrm{H}, 2 \times \mathrm{CH}_{3} \mathrm{O}\right) .3 .89\left(\mathrm{~s}, 3 \mathrm{H}, \mathrm{CH}_{3} \mathrm{O}\right), 6.48\left(\mathrm{~s}, 2 \mathrm{H}, \mathrm{H}-2^{\prime}, 6^{\prime}\right)$, 7.38 (dd, 1H, $J=8.6,0.9 \mathrm{~Hz}, \mathrm{H}-10), 7.43$ (ddd, 1H, $J=8.0,0.9 \mathrm{~Hz}, \mathrm{H}-12), 7.46$ (d, $1 \mathrm{H}, J=0.7$ Hz, H-6), 7.67 (ddd, 1H, J=8.6, 7.0, 1.6 Hz, H-11), 8.18 (d, 1H, J=0.7 Hz, H-4), 8.27 (dd, 1H, $J=8.0,1.6 \mathrm{~Hz}, \mathrm{H}-13)$, OH not seen.

\section{General procedures 5-hydroxy-3-( $\mathbf{R}^{1}$-phenyl)-2H,10aH-pyrano[2,3-b]chromen-2-ones (5a- 5c)}

Method F. Compound 3a (10.0 mmol) was dissolved in acetic acid $(10 \mathrm{~mL})$ and the solution was stirred at $60{ }^{\circ} \mathrm{C}$ for $1 \mathrm{~h}$. After cooling the yellow precipitate was filtered off, washed with diethyl ether and crystallized from toluene or acetic acid.

Method G. Compounds 5 as the side products were isolated by flash chromatography from the filtrate obtained after products $\mathbf{3}$ isolation (see Method C).

5-Hydroxy-3-phenyl-2H,10aH-pyrano[2,3-b]chromen-2-one (5a). Method F, work-up as described above gave $77 \%$ of $\mathbf{5 a}\left(\operatorname{method} \mathrm{G}, 2 \%\right.$ ) as yellow powder: mp $256-258{ }^{\circ} \mathrm{C} . \mathrm{R}_{\mathrm{f}}=0.62$ in EtOAc/hexane 1:1. Anal. calcd. for $\mathrm{C}_{18} \mathrm{H}_{12} \mathrm{O}_{4}$ (292.3): C, 73.97; H, 4.14. Found: C, 74.18; $\mathrm{H}, 4.16$. IR $v(\mathrm{KBr}): 3450,3060,1731,1715,1645,1610,1557,1489,1460,1371,1355,1316$, 1296, 1271, 1233, 1204, 1180, 1113, 1037, 1019, 951, 912, 785, 759, $737 \mathrm{~cm}^{-1} .{ }^{1} \mathrm{H}$ NMR (300 $\left.\mathrm{MHz}, \mathrm{DMSO}-d_{6}\right) \delta 6.73(1 \mathrm{H}, \mathrm{s}, \mathrm{H}-10 \mathrm{a}), 7.15(1 \mathrm{H}, \mathrm{s}, \mathrm{H}-4), 7.17(1 \mathrm{H}, \mathrm{dd}, J=8.2,1.0 \mathrm{~Hz}, \mathrm{H}-9)$, $7.21(1 \mathrm{H}, \mathrm{ddd}, J=7.6,7.4,1.0 \mathrm{~Hz}, \mathrm{H}-7), 7.35-7.41(3 \mathrm{H}, \mathrm{m}, \mathrm{H}-3$ ', 4', 5'), 7.42 (1H, ddd, $J=8.2$, 7.1, $1.5 \mathrm{~Hz}, \mathrm{H}-8), 7.47-7.51$ (2H, m, H-2', 6'), 7.89 (1H, dd, $J=7.7,1.5 \mathrm{~Hz}, \mathrm{H}-6), 8.12(1 \mathrm{H}, \mathrm{s}$, $\mathrm{OH}) .{ }^{13} \mathrm{C}$ NMR $\left(75 \mathrm{MHz}, \mathrm{DMSO}-d_{6}\right) \delta 30.7,96.9,107.5,114.3,117.7,122.1,122.6,122.8$, $125.0,128.0,128.3,128.6,132.5,134.4,139.4,150.3,151.8,159.4$.

3-(4-Fluorophenyl)-5-hydroxy-2H,10aH-pyrano[2,3-b]chromen-2-one (5b). Method G, workup as described above gave $4 \%$ of $\mathbf{5 b}$, as yellow powder: $\mathrm{mp} 260-262{ }^{\circ} \mathrm{C} . \mathrm{R}_{\mathrm{f}}=0.52$ in EtOAc/hexane 1:1. Anal. calcd. for $\mathrm{C}_{18} \mathrm{H}_{11} \mathrm{FO}_{4}$ (310.3): C, 69.68; H, 3.57. Found: C, 69.44; H, 3.79. IR $v$ (KBr): $3450,1730,1715,1645,1615,1557,1485,1375,1355,1298,1271,1230$, 1204, 1180, 1040, 1025, 951, 918, $785 \mathrm{~cm}^{-1} .{ }^{1} \mathrm{H}$ NMR (300 MHz, DMSO-d $) \delta 6.90(\mathrm{~s}, 1 \mathrm{H}, \mathrm{H}-$ 10a), 7.23 (ddd, 1H, $J=7.7,7.4,1.1 \mathrm{~Hz}, \mathrm{H}-7), 7.28$ (dd, 1H, $J=8.3,1.1 \mathrm{~Hz}, \mathrm{H}-9), 7.24-7.33$ (m, 2H, H-3',5'), 7.53 (ddd, 1H, $J=8.3,7.4,1.5 \mathrm{~Hz}, \mathrm{H}-8), 7.56$ (s, 1H, H-4), 7.58-7.65 (m, 2H, H-2', 6'), $7.72(\mathrm{dd}, 1 \mathrm{H}, J=7.7,1.5 \mathrm{~Hz}, \mathrm{H}-6)$, OH not seen.

5-Hydroxy-3-(4-methoxyphenyl)-2H,10aH-pyrano[2,3-b]chromen-2-one (5c). Method G, work-up as described above gave $2 \%$ of $\mathbf{5 b}$, as yellow powder: mp $260-262{ }^{\circ} \mathrm{C}$. $\mathrm{R}_{\mathrm{f}}=0.34$ in EtOAc/hexane 1:1. Anal. calcd. for $\mathrm{C}_{19} \mathrm{H}_{14} \mathrm{O}_{5}$ (322.3): C, 70.80; H, 4.38. Found: 70.63; H, 4.52. IR $v(\mathrm{KBr}): 3440,1728,1720,1651,1615,1559,1480,1375,1358,1292,1277,1206,1188$, $1025,955,919,780 \mathrm{~cm}^{-1} .{ }^{1} \mathrm{H}$ NMR $\left(300 \mathrm{MHz}, \mathrm{DMSO}-d_{6}\right) \delta 3.81\left(\mathrm{~s}, 3 \mathrm{H}, \mathrm{CH}_{3} \mathrm{O}\right), 6.90(\mathrm{~s}, 1 \mathrm{H}, \mathrm{H}-$ 10a), 7.01-7.03 (m, 2H, H-3',5'), 7.23 (ddm, 1H, $J=7.6,4.4$ Hz, H-7), 7.30-7.32 (m, 1H, H-9), 
7.50 (s, 1H, H-4), 7.45-7.60 (m, 3H, H-8, 2',6' ), 7.70-7.74 (m, 1H, H-6), OH not seen. ${ }^{13} \mathrm{C}$ NMR $\left(300 \mathrm{MHz}, \mathrm{DMSO}-d_{6}\right) \delta 55.2,93.1,107.2,113.8,114.6,117.6,122.3,123.1,124.8,126.3,129.2$, $132.5,137.0,149.8,151.3,159.5,159.6$.

\section{General procedures for 5-hydroxy-3-( $\mathbf{R}^{1}$-phenyl)-2H,5H-pyrano[3,2-c]chromen-2-ones (6a-} 6f)

Method H. A mixture of acetate $3(0.41 \mathrm{mmol}, 1.00 \mathrm{~mol} \mathrm{eq})$ and p-toluenesulfonic acid (15 mg, $0.09 \mathrm{mmol}, 0.22 \mathrm{~mol} \mathrm{eq})$ was heated in $50 \mathrm{~mL}$ of mixture dioxane-water $(3: 1)$ at $50{ }^{\circ} \mathrm{C}$ for $2 \mathrm{~h}$. After cooling the solid product was filtered off, washed with cold diethyl ether and crystallized from dioxane.

Method I. A mixture of acetate $3(0.41 \mathrm{mmol}, 1.00 \mathrm{~mol} \mathrm{eq})$, and water $(0.5 \mathrm{~mL}, 27.8 \mathrm{mmol}$, $67.80 \mathrm{~mol} \mathrm{eq}$ ) in $0.5 \mathrm{~mL}$ of THF was stirred at room temperature for $15 \mathrm{~h}$. Then the mixture was diluted with ethyl acetate $(20 \mathrm{~mL})$ and extracted with water $(3 \times 20 \mathrm{~mL})$. The organic layer was dried with $\mathrm{Na}_{2} \mathrm{SO}_{4}$. After filtration and evaporation of ethyl acetate, the solid product was crystallized from dioxane.

5-Hydroxy-3-phenyl-2H,5H-pyrano[3,2-c]chromen-2-one (6a). Method $H$, work-up as described above gave $74 \%$ of $\mathbf{6 a}$ as yellow powder: mp $150-151{ }^{\circ} \mathrm{C}$ (dioxane), $\mathrm{R}_{\mathrm{f}}=0.62$ in EtOAc/hexane 1:1. Anal. calcd. for $\mathrm{C}_{18} \mathrm{H}_{12} \mathrm{O}_{4}$ (292.3): C, 73.97; H, 4.14. Found: C, 74.19; $\mathrm{H}, 4.13$. IR $v(\mathrm{KBr}): 3381,3030,1760,1705,1639,1610,1546,1464,1371,1352,1220,1191$, $1175,1042,1018,973,933,899,758,696 \mathrm{~cm}^{-1} .{ }^{1} \mathrm{H}$ NMR $\left(300 \mathrm{MHz}, \mathrm{CDCl}_{3}\right) \delta 3.51(1 \mathrm{H}, \mathrm{d}, J=$ $6.4 \mathrm{~Hz}, \mathrm{OH}), 6.43(1 \mathrm{H}, \mathrm{d}, J=6.6 \mathrm{~Hz}, \mathrm{H}-5), 7.07(1 \mathrm{H}, \mathrm{dd}, J=8.1,1.0 \mathrm{~Hz}, \mathrm{H}-7), 7.13(1 \mathrm{H}, \mathrm{ddd}, J$ $=8.1,1.0 \mathrm{~Hz}, \mathrm{H}-8), 7.39-7.44$ (4H, m, H-9,3',4',5'), 7.49 (1H, s, H-4), 7.66-7.71 (2H, m, H$\left.2^{\prime}, 6^{\prime}\right), 7.87(1 \mathrm{H}, \mathrm{dd}, J=7.8,1.7 \mathrm{~Hz}, \mathrm{H}-10) .{ }^{13} \mathrm{C}$ NMR $\left(75 \mathrm{MHz}, \mathrm{DMSO}-d_{6}\right) \delta 91.1,109.6,114.4$, $117.5,121.9,122.0,124.5,124.8,125.2,127.9,128.2,132.3,134.4,134.8,139.7,152.6,159.7$, 168.3 .

3-(4-Fluorophenyl)-5-hydroxy-2H,5H-pyrano[3,2-c]chromen-2-one (6b). Method I, work-up as described above gave $78 \%$ of $\mathbf{6 b}$ as yellow powder: mp $290{ }^{\circ} \mathrm{C}$ dec., $\mathrm{R}_{\mathrm{f}}=0.52$ in EtOAc/hexane 1:1. Anal. calcd. for $\mathrm{C}_{18} \mathrm{H}_{11} \mathrm{FO}_{4}$ (310.3): C, 69.68; H, 3.57. Found: C, 69.41; H, 3.36. IR $v$ (nujol): $3388,1702,1636,1548,1301,1266,1156,1038,983,840,761 \mathrm{~cm}^{-1} .{ }^{1} \mathrm{H}$ NMR (300 MHz, DMSO- $\left.d_{6}\right) \delta 6.43(\mathrm{~d}, 1 \mathrm{H}, J=6.6 \mathrm{~Hz}, \mathrm{H}-5), 7.12$ (dd, $\left.1 \mathrm{H}, J=8.3,1.1 \mathrm{~Hz}, \mathrm{H}-7\right)$, 7.17 (ddd, $1 \mathrm{H}, J=7.8,7.3,1.1 \mathrm{~Hz}, \mathrm{H}-9), 7.23-7.36$ (m, 2H, H-3',5'), 7.47 (ddd, $1 \mathrm{H}, J=8.3,7.3$, $1.7 \mathrm{~Hz}, \mathrm{H}-8), 7.73$ (dd, 1H, $J=7.8,1.7 \mathrm{~Hz}, \mathrm{H}-10), 7.74$ (d, 1H, $J=6.6 \mathrm{~Hz}, \mathrm{H}-7), 7.77-7.83$ (m, $\left.2 \mathrm{H}, \mathrm{H}-2^{\prime}, 6^{\prime}\right), 7.88$ (s, 1H, H-4). ${ }^{13} \mathrm{C}$ NMR (300 MHz, DMSO-d $) \delta 91.2,109.7,114.4,115.2$, 117.6, 122.1, 122.2, 123.7, 130.2, 130.9, 132.4, 139.7, 150.1, 152.7, 159.8, 162.1.

5-Hydroxy-3-(4-methoxyphenyl)-2H,5H-pyrano[3,2-c]chromen-2-one (6c). Method I, workup as described above gave $98 \%$ of $\mathbf{6 c}$ as yellow powder: $\mathrm{mp} 235-238{ }^{\circ} \mathrm{C}, \mathrm{R}_{\mathrm{f}}=0.34$ in EtOAc/hexane 1:1. Anal. calcd. for $\mathrm{C}_{19} \mathrm{H}_{14} \mathrm{O}_{5}$ (322.3): C, 70.80; H, 4.38. Found: C, 70.59; H, 4.09. IR $v$ (nujol): $3346,1725,1645,1602,1552,1513,1288,1245,1184,1113,1044,1021$, 979, 902, 879, 842, 767, 735, $687 \mathrm{~cm}^{-1} .{ }^{1} \mathrm{H}$ NMR (300 MHz, DMSO- $\left.d_{6}\right) \delta 3.81\left(\mathrm{~s}, 3 \mathrm{H}, \mathrm{CH}_{3} \mathrm{O}\right)$, $6.42(\mathrm{~d}, 1 \mathrm{H}, J=6.6 \mathrm{~Hz}, \mathrm{H}-5), 6.99-7.05$ (m, 2H, H-3',5'), 7.12 (dd, 1H, $J=8.3,1.1 \mathrm{~Hz}, \mathrm{H}-7$ ), 
7.17 (ddd, $1 \mathrm{H}, J=7.8,7.4,1.1 \mathrm{~Hz}, \mathrm{H}-9), 7.46$ (ddd, 1H, $J=8.3,7.4,1.7 \mathrm{~Hz}, \mathrm{H}-8), 7.72$ (dd, 1H, $J=7.8,1.1 \mathrm{~Hz}, \mathrm{H}-10), 7.72$ (d, 1H, $J=6.6 \mathrm{~Hz}, \mathrm{H}-7), 7.69-7.76$ (m, 2H, H-2',6'), 7.80 (s, 1H, H4). ${ }^{13} \mathrm{C}$ NMR (300 MHz, DMSO- $\left.d_{6}\right) \delta 55.1,91.2,109.7,113.7,113.7,114.5,117.4,121.9,121.9$, $124.2,126.6,129.2,129.2,132.1,138.1,149.2,152.4,159.4,159.8$.

5-Hydroxy-3-(3,4,5-trimethoxyphenyl)-2H,5H-pyrano[3,2-c]chromen-2-one (6d). Method I, work-up as described above gave $80 \%$ of $\mathbf{6 d}$ as yellow powder: $\mathrm{mp} 219-221{ }^{\circ} \mathrm{C}, \mathrm{R}_{\mathrm{f}}=0.21$ in EtOAc/hexane 1:1. Anal. calcd. for $\mathrm{C}_{21} \mathrm{H}_{18} \mathrm{O}_{7}$ (382.4): C, 65.96; H, 4.74. Found: C, 65.67; H, 4.44. IR $v$ (nujol): $3432,1725,1645,1587,1561,1332,1294,1233,1121,1041,999,877,821$, $764 \mathrm{~cm}^{-1} .{ }^{1} \mathrm{H}$ NMR (300 MHz, DMSO- $\left.d_{6}\right) \delta 3.71\left(\mathrm{~s}, 3 \mathrm{H}, \mathrm{CH}_{3} \mathrm{O}\right), 3.83\left(\mathrm{~s}, 6 \mathrm{H}, \mathrm{CH}_{3} \mathrm{O}\right), 6.42(\mathrm{~d}$, $1 \mathrm{H}, J=6.6 \mathrm{~Hz}, \mathrm{H}-5), 7.08$ (s, 2H, H-2',6'), 7.13 (dd, 1H, $J=8.3,1.0 \mathrm{~Hz}, \mathrm{H}-7$ ), 7.18 (ddd, 1H, $J=$ 7.9, 7.4, 1.0 Hz, H-9), 7.48 (ddd, $1 \mathrm{H}, J=8.3,7.4,1.0 \mathrm{~Hz}, \mathrm{H}-8$ ), 7.72 (dd, 1H, J= 7.9, 1.6 Hz, H10), $7.75(\mathrm{~d}, 1 \mathrm{H}, J=6.6 \mathrm{~Hz}, \mathrm{H}-7), 7.93$ (s, 1H, H-4). ${ }^{13} \mathrm{C}$ NMR (75 MHz, DMSO- $\left.d_{6}\right) \delta 56.0$, $56.0,60.1,91.3,105.7,109.7,114.5,117.6,122.1,124.4,129.9,132.4,137.9,137.9,139.5$, $149.8,152.5,152.6,159.7$.

5-Hydroxy-3-(4-nitrophenyl)-2H,5H-pyrano[3,2-c]chromen-2-one (6e). Method I, work-up as described above gave $70 \%$ of $6 \mathbf{e}$ as yellow powder: $\mathrm{mp} 192-195{ }^{\circ} \mathrm{C}, \mathrm{R}_{\mathrm{f}}=0.24$ in EtOAc/hexane 1:1. Anal. calcd. for $\mathrm{C}_{18} \mathrm{H}_{11} \mathrm{NO}_{6}$ (337.3): C, 64.10; H, 3.29; N, 4.15. Found: C, 63.63; H, 3.57; N, 4.28. IR $v$ (nujol): 3538, 1737, 1698, 1636, 1546, 1518, 1346, 1325, 1221, 1156, 1039, 974, 855, $776 \mathrm{~cm}^{-1} .{ }^{1} \mathrm{H}$ NMR $\left(300 \mathrm{MHz}, \mathrm{DMSO}-d_{6}\right) \delta 6.46(\mathrm{~d}, 1 \mathrm{H}, J=6.6 \mathrm{~Hz}, \mathrm{H}-5), 7.14(\mathrm{dd}, 1 \mathrm{H}, J=8.3$, $0.9 \mathrm{~Hz}, \mathrm{H}-7), 7.19$ (ddd, $1 \mathrm{H}, J=7.9,7.4,0.9 \mathrm{~Hz}, \mathrm{H}-9), 7.51$ (ddd, $1 \mathrm{H}, J=8.3,7.4,1.5 \mathrm{~Hz}, \mathrm{H}-8$ ), $7.76(\mathrm{dd}, 1 \mathrm{H}, J=7.9,1.5 \mathrm{~Hz}, \mathrm{H}-10), 7.82$ (d, 1H, $J=6.6 \mathrm{~Hz}, \mathrm{OH}), 8.02-8.08$ (m, 2H, H-2',6' ), 8.12 (s, 1H, H-4), 8.28-8.34 (m, 2H, H-3',5'). ${ }^{13} \mathrm{C}$ NMR (75 MHz, DMSO- $\left.d_{6}\right) \delta$ 91.2, 109.7, $114.3,117.7,123.5,122.2,122.5,129.2,130.2,133.0,141.1,142.0,146.9,151.5,153.0,159.3$.

5-Hydroxy-3-[(4-methylsulfonyl)phenyl]-2H,5H-pyrano[3,2-c]chromen-2-one (6f). Method I, work-up as described above gave $26 \%$ of $6 \mathbf{f}$ as yellow powder: mp $181-183{ }^{\circ} \mathrm{C}, \mathrm{R}_{\mathrm{f}}=0.31$ in EtOAc/hexane 1:1. Anal. calcd. for $\mathrm{C}_{19} \mathrm{H}_{14} \mathrm{O}_{6} \mathrm{~S}$ (370.1): C, 61.61; H, 3.81; S, 8.66. Found: C, 61.49; H, 3.51; S, 8.29. IR v(nujol): 3424, 1729, 1644, 1604, 1593, 1553, 1486, 1409, 1296, 1270, 1203, 1142, 1090, 1041, 988, 958, 845, $766 \mathrm{~cm}^{-1} .{ }^{1} \mathrm{H}$ NMR (300 MHz, DMSO- $\left.d_{6}\right) \delta 3.26$ (s, 3H, $\left.\mathrm{SO}_{2} \mathrm{CH}_{3}\right), 7.14$ (d, 1H, $\left.J=7.0 \mathrm{~Hz}, \mathrm{H}-5\right), 7.19$ (ddd, $1 \mathrm{H}, J=7.7,7.3,1.0 \mathrm{~Hz}, \mathrm{H}-9$ ), 7.50 (ddd, $1 \mathrm{H}, J=8.3,7.4,1.6 \mathrm{~Hz}, \mathrm{H}-8), 7.76$ (dd, 1H, $J=7.7,1.6 \mathrm{~Hz}, \mathrm{H}-10), 7.80$ (d, 1H, $J=7.0 \mathrm{~Hz}$, H-7), 7.96-8.02 (m, 4H, H-2',3',5',6'), 8.05 (s, 1H, H-4). ${ }^{13} \mathrm{C}$ NMR (75 MHz, DMSO- $\left.d_{6}\right) \delta 43.5$, $91.2,109.7,114.3,117.7,122.2,122.4,122.8,126.9,128.9,132.9,139.4,140.1,141.5,151.2$, $152.9,159.5$.

General procedures for 3-( $\mathbf{R}^{1}$-phenyl)-5-( $\mathbf{R}^{3}$-oxy)-2H,5H-pyrano[3,2-c]chromen-2-ones (7a7d)

Method J: A solution of acetate $3(3.00 \mathrm{mmol})$ and p-toluenesulfonic acid $(8.0 \mathrm{mg}, 0.04 \mathrm{mmol})$ in prop-2-yn-1-ol $(10 \mathrm{~mL})$ was heated at $60{ }^{\circ} \mathrm{C}$ for $3 \mathrm{~h}$. After cooling, $30 \mathrm{~mL}$ of diethyl ether was added, the formed precipitate filtered off, washed with diethyl ether and crystallized from dioxane. 
Method K: A solution of acetate $3(2.00 \mathrm{mmol})$ and p-toluenesulfonic acid (5.0 $\mathrm{mg}, 0.03 \mathrm{mmol})$ in prop-2-yn-1-ol (4 mL) was irradiated in microwave oven at $60{ }^{\circ} \mathrm{C}$ and $10 \mathrm{~W}$ for $10 \mathrm{~min}$. The products 7 were isolated and purified in the same manner as described in Method A.

3-Phenyl-5-(prop-2-yn-1-yloxy)-2H,5H-pyrano[3,2-c]chromen-2-one (7a). Method J, workup as described above gave $70 \%$ of $7 \mathbf{a}$ (method $\mathrm{K}, 75 \%$ ) as yellow powder: $\mathrm{mp} 185-188{ }^{\circ} \mathrm{C}$. Anal. calcd. for $\mathrm{C}_{21} \mathrm{H}_{14} \mathrm{O}_{4}$ (330.4): C, 76.35; H, 4.27. Found: C, 76.59; H, 4.46. IR $v$ (nujol): $1718,1710,1652,1565,1488,1465,1297,1190,1042,1017,981,897,785,680 \mathrm{~cm}^{-1}$. ${ }^{1} \mathrm{H}$ NMR $\left(300 \mathrm{MHz}, \mathrm{DMSO}-d_{6}\right) \delta 3.56(\mathrm{t}, 1 \mathrm{H}, J=2.4 \mathrm{~Hz}, \mathrm{CH}), 4.47\left(\mathrm{~d}, 2 \mathrm{H}, J=2.4 \mathrm{~Hz}, \mathrm{CH}_{2}\right), 6.41(\mathrm{~s}, 1 \mathrm{H}$, H-5), 7.24 (ddd, 1H, J=7.7, 7.1, 0.9 Hz, H-9), 7.53 (ddd, 1H, J=8.3, 7.2, $1.6 \mathrm{~Hz}, \mathrm{H}-8$ ), 7.72 (d, $1 \mathrm{H}, J=7.5 \mathrm{~Hz}, \mathrm{H}-7), 7.73\left(\mathrm{ddd}, 2 \mathrm{H}, J=7.9,7.1,1.2 \mathrm{~Hz}, \mathrm{H}-2^{\prime}, 6^{\prime}\right), 7.76$ (dd, $1 \mathrm{H}, J=8.2,1.8 \mathrm{~Hz}$, H-10), 7.73-7.84 (m, 3H, H-3',4',5'), 7.87 (s, 1H, H-4).

3-(4-Methoxyphenyl)-5-(prop-2-yn-1-yloxy)-2H,5H-pyrano[3,2-c]chromen-2-one (7b). Method J, work-up as described above gave $74 \%$ of $7 \mathbf{b}$ (method $\mathrm{K}, 77 \%$ ) as yellow powder: mp 202-205 ${ }^{\circ} \mathrm{C}$. Anal. calcd. for $\mathrm{C}_{22} \mathrm{H}_{16} \mathrm{O}_{5}$ (360.4): C, 73.33; H, 4.48. Found: C, 73.54; H, 4.57. IR v(nujol): $1718,1707,1652,1565,1480,1464,1298,1190,1050,1018,976,898,788,675 \mathrm{~cm}^{-1}$. ${ }^{1} \mathrm{H}$ NMR $\left(300 \mathrm{MHz}, \mathrm{DMSO}-d_{6}\right) \delta 3.55(\mathrm{t}, 1 \mathrm{H}, J=2.4 \mathrm{~Hz}, \mathrm{CH}), 4.46\left(\mathrm{~d}, 1 \mathrm{H}, J=2.4 \mathrm{~Hz}, \mathrm{CH}_{2}\right), 6.39(\mathrm{~s}, 1 \mathrm{H}$, H-5), 7.03 (ddd, 2H, H-3',5'), 7.22 (d, 1H, $J=8.0$ Hz, H-7), 7.24 (ddd, 1H, J= 7.7, 1.1 Hz, H-9), $7.51(\mathrm{ddd}, 1 \mathrm{H}, J=8.0,1.6 \mathrm{~Hz}, \mathrm{H}-8), 7.71\left(\mathrm{ddd}, 2 \mathrm{H}, J=6.9,2.1 \mathrm{~Hz}, \mathrm{H}-2^{\prime}, 6^{\prime}\right), 7.75$ (dd, 1H, $J=$ 7.7, 1.6 Hz, H-10), 7.79 (s, 1H, H-4).

3-(4-Nitrophenyl)-5-(prop-2-yn-1-yloxy)-2H,5H-pyrano[3,2-c]chromen-2-one (7c). Method J, work-up as described above gave $65 \%$ of $7 \mathrm{c}$ (method $\mathrm{K}, 68 \%$ ) as yellow powder: $\mathrm{mp} 232-235$ ${ }^{\circ} \mathrm{C}$. Anal. calcd. for $\mathrm{C}_{21} \mathrm{H}_{13} \mathrm{NO}_{6}$ (375.3): C, 67.20; H, 3.49; N, 3.73. Found: C, 67.51; H, 3.58; N, 3.99. IR $v$ (nujol): 1720, 1710, 1645, 1560, 1485, 1460, 1295, 1197, 1045, 1015, 975, 900, 788, $672 \mathrm{~cm}^{-1} .{ }^{1} \mathrm{H}$ NMR (300 MHz, DMSO-d $) \delta 3.57(\mathrm{t}, 1 \mathrm{H}, J=2.4 \mathrm{~Hz}, \mathrm{CH}), 4.48(\mathrm{~d}, 2 \mathrm{H}, J=2.4 \mathrm{~Hz}$, $\mathrm{CH}_{2}$ ), 6.42 (s, 1H, H-5), 7.26 (d, 1H, J=7.7 Hz, H-7), 7.28 (ddd, 1H, J=7.8, $0.9 \mathrm{~Hz}, \mathrm{H}-9$ ), 7.56 (ddd, 1H, $J=7.8,1.2 \mathrm{~Hz}, \mathrm{H}-8), 7.80$ (dd, 1H, $J=7.8,1.2 \mathrm{~Hz}, \mathrm{H}-10), 8.06$ (ddd, 2H, $J=9.0,2.0$ Hz, H-2',6' ), 8.10 (s, 1H, H-4), 8.32 (ddd, 2H, $J=9.0,2.0$ Hz, H-3',5').

5-[(2-Hydroxyethyl)thio]-3-phenyl-2H,5H-pyrano[3,2-c]chromen-2-one (7d). A solution of acetate $3 \mathbf{a}(1.10 \mathrm{~g}, 3.00 \mathrm{mmol}$,), 2-thioethanol $(0.25 \mathrm{~mL}, 1.10 \mathrm{~mol} \mathrm{eq})$ and p-toluenesulfonic acid ( $8.0 \mathrm{mg}, 0.04 \mathrm{mmol})$ in $\mathrm{CH}_{3} \mathrm{NO}_{2}(3 \mathrm{~mL})$ was stirred and heated at $60{ }^{\circ} \mathrm{C}$ for $3 \mathrm{~h}$. After cooling, $30 \mathrm{~mL}$ of diethyl ether was added, the formed solid was filtered off, washed with diethyl ether and crystallized from dioxane to give $75 \%$ of $7 \mathbf{d}$ as yellow powder: mp $184-186{ }^{\circ} \mathrm{C}$. Anal. calcd. for $\mathrm{C}_{20} \mathrm{H}_{16} \mathrm{O}_{4} \mathrm{~S}$ (352.4): C, 68.16; H, 4.58; S, 9.10. Found: C, 68.41; H, 4.43; S, 9.22. IR v (nujol): $1716,1648,1615,1555,1480,1295,1260,1190,1145,1070,1042,978,912,895,771,689 \mathrm{~cm}^{-}$ 1. ${ }^{1} \mathrm{H}$ NMR (300 MHz, DMSO- $\left.d_{6}\right) \delta$ 2.73-2.93 (m, 2H, $\left.\mathrm{CH}_{2} \mathrm{O}\right), 3.40(\mathrm{br}, 1 \mathrm{H}, \mathrm{OH}), 3.49-3.74(\mathrm{~m}$, 2H, $\mathrm{CH}_{2} \mathrm{~S}$ ), 6.97 (s, 1H, H-5), 7.11 (d, 1H, J=8.1 Hz, H-7), 7.12 (ddd, 1H, J=7.7, 0.5 Hz, H-9), 7.21(ddd, $1 \mathrm{H}, J=8.1,1.5 \mathrm{~Hz}, \mathrm{H}-8), 7.41-7.51$ (m, 3H, H-10, 2',6'), 7.69-7.71 (m, 3H, H-3',4',5'), 7.78 (s, 1H, H-4).

5-Ethoxy-3-(4-fluorophenyl)-2H,10aH-pyrano[2,3-b]chromen-2-one (8). Compound 8 was isolated in $5 \%$ (from the crude reaction mixture obtained by synthesis of $\mathbf{5 b}$ ) by flash 
chromatography in ethyl acetate/hexane (1:2) as yellow powder: $\mathrm{mp} 160-164{ }^{\circ} \mathrm{C}, \mathrm{R}_{\mathrm{f}}=0.66$ in EtOAc/hexane 1:2. Anal. calcd. for $\mathrm{C}_{20} \mathrm{H}_{15} \mathrm{FO}_{4}$ (338.3): C, 71.00; H, 4.47. Found: C, 71.18; H, 4.33. IR $v$ (KBr): 1717, 1644, 1605, 1555, 1509, 1462, 1370, 1262, 1231, 1157, 1069, 1022, 968, 907, 841, $760 \mathrm{~cm}^{-1} .{ }^{1} \mathrm{H}$ NMR (300 MHz, DMSO- $\left.d_{6}\right) \delta 1.13\left(\mathrm{dd}, 3 \mathrm{H}, J=7.0 \mathrm{~Hz}, \mathrm{CH}_{3}\right), 3.78(\mathrm{dq}$, $1 \mathrm{H}, J=9.8,6.9 \mathrm{~Hz}, \mathrm{CH}_{2}$ ), 3.89 (dq, $\left.1 \mathrm{H}, J=9.8,7.0 \mathrm{~Hz}, \mathrm{CH}_{2}\right), 6.29$ (s, 1H, H-10a), 7.19 (dd, 1H, $J=8.3,0.9 \mathrm{~Hz}, \mathrm{H}-9), 7.22$ (ddd, 1H, $J=7.7,7.5,0.9 \mathrm{~Hz}, \mathrm{H}-7), 7.35-7.27$ (m, 2H, H-3', 5'), 7.51 (ddd, $1 \mathrm{H}, J=8.3,7.5,1.5 \mathrm{~Hz}, \mathrm{H}-8), 7.75$ (dd, 1H, $J=7.7,1.5 \mathrm{~Hz}, \mathrm{H}-6), 7.84-7.55$ (m, 2H, H$\left.2^{\prime}, 6^{\prime}\right), 7.92(\mathrm{~s}, 1 \mathrm{H}, \mathrm{H}-4)$.

\section{Acknowledgements}

This work was supported by the Slovak Research Agency under the contract No. VEGA 1_0448_09, LPP-0153-06, Biomagi Ltd. and disseminated by COST Action CM0602. The authors are appreciated the members of Developmental Therapeutics Program of National Cancer Institute, Bethesda, Maryland USA for anticancer activity screening.

\section{References}

1. Ghosh, C. K. Heterocycles 2004, 63, 2875.

2. Ishar, M. P. S.; Singh, G.; Singh, S.; Sreenivasan, K. K.; Singh, G. Bioorg. Med. Chem. Lett. 2006, 16, 1366.

3. Nawrot-Modranka, J.; Nawrot, E.; Graczyk, J. Eur. J. Med. Chem. 2006, 41, 1301.

4. El-Shaaer, H. M.; Foltínová, P.; Lácová, M.; Chovancová, J.; Stankovičová, H. Farmaco 1998, 53, 224.

5. Kirkiacharian, S.; Thuy, D. T.; Sicsic, S.; Bakhchinian, R.; Kurkjian, R.; Tonnaire, T. Farmaco 2002, 57, 703.

6. Rakowitz, D.; Gmeiner, A.; Schröder, N.; Matuszczak, B. Eur. J. Parm. Sci. 2006, 27, 188.

7. Santini, C.; Berger, G. D.; Han, W.; Mosley, R.; MacNaul, K.; Berger, J.; Doebber, T.; Wu, M.; Moller, D. E.; Tolman, R. L.; Sahoo, S. P. Bioorg. Med. Chem. Lett. 2003, 13, 1277.

8. Silva, V. L. M.; Silva, A. M. S.; Pinto, D. C. G. A.; Cavaleiro, J. A. S.; Patonay, T. Synlett 2004, 2717.

9. Gašparová, R.; M. Lácová, M.; Krutošíková, A. Collect. Czech. Chem. Commun. 2005, 70 2101.

10. Stankovičová, H.; Gáplovský, A.; Lácová, M.; Chovancová, J.; Puchala, A. J. Heterocycl. Chem. 2006, 43, 843.

11. Gáplovský, A.; Donovalová, J.; Lácová, M.; Mračnová, R.; El-Shaaer, H. M. J. Photochem. Photobiol., A 2000, 136, 61.

12. Stankovičová, H.; Fabian, W. M. F.; Lácová, M. Molecules 1997, 1, 223. 
13. Ghosh, C. K.; Bandyopadhyay, C. Indian J. Chem., Sect. B 1984, 23, 1048.

14. Ghosh, C. K.; Bandyopadhyay, C.; Biswas, S.; Chandravarty, A. K. Indian J. Chem., Sect. B 1990, 29, 814.

15. Lácová, M.; Stankovičová, H.; Boháč, A.; Kotzianová, B. Tetrahedron 2008, 64, 9646.

16. Lácová, M.; Gašparová, R.; Koiš, P.; Boháč, A.; El-Shaaer, H. M. Tetrahedron 2010, 66, 1410.

17. http://dtp.nci.nih.gov/screening.html

18. http://dtp.nci.nih.gov/compare/

19. http://dtp.nci.nih.gov/docs/compare/examples/antitub.html

20. Lácová, M.; Boháč, A. Patent Application No. PP5006 - 2006.

21. Nohara A.; Umetani T.; Sanno Y. Tetrahedron 1974, 30, 3553.

22. https://dtps7.ncifcrf.gov/ 